\title{
Up-Regulated Expression of MCM7 is a Poor Prognostic Factor in Colorectal Cancer
}

\author{
Ting Wang ${ }^{1}$, Bing Xu ${ }^{1}$, Jing Zhang ${ }^{1}$, Ying Zheng ${ }^{1}$, Suzhen Yang ${ }^{1}$ and Lei Dong ${ }^{1 *}$ \\ ${ }^{1}$ Department of Digestive Disease and Gastrointestinal Motility Research Room, The second Affiliated Hospital of Xi'an Jiaotong University, \\ Xi'an, Shaanxi, MI 710004, PR China
}

Received: January 28, 2019; Accepted: February 07, 2019; Published: February 13, 2019

*Corresponding author: Professor Lei Dong, Department of Digestive Disease and Gastrointestinal Motility Research Room, The second Affiliated Hospital of Xi'an Jiaotong University, 157 West Fifth Road, Xi'an, Shaanxi Province, 710004, P.R. China, Email: dong556@126.com

\begin{abstract}
Objective: Colorectal cancer (CRC) is one of the most common malignancies worldwide. MCM7 is one of the important components of DNA replication complex involved in cancer progression. At present, the significance of MCM7 and its mechanisms in CRC development are still unclear.

Materials and Methods: Gene expression data of CRC was downloaded from Gene Expression Omnibus (GEO) and TCGA databases. Bioinformatics method was applied to screen CRC differentially expressed genes (DEGs). MCODE and degree value analysis were used to select core genes. The datasets were explored to verify the mRNA expression level of MCM7, and the protein expression level of MCM7 was further verified by Western Blotting and immunohistochemical analysis. Moreover, we also analyzed the correlation between MCM7 and clinical characteristics. The prognostic value of MCM7 in CRC was explored through Kaplan-Meier analysis, univariate and multivariate Cox regression analysis.
\end{abstract}

Results: We confirmed that MCM7 was up-regulated in CRC tissues compared with adjacent non-tumor tissues. MCM7 overexpression was positively associated with lymph node metastasis and TNM stage in CRC. Higher expression of MCM7 showed shorter overall survival rate. Furthermore, MCM7 level could be an independent prognostic factor for CRC.

Conclusion: Our study suggested that MCM7 may be a new prognostic factor or molecular target in CRC. Values

Key Words: Colorectal Cancer; Bioinformatics; MCM7; Prognostic

\section{Introduction}

Colorectal cancer (CRC) is one of the most common malignancies in the digestive system, which ranks the third most common cancer in both men and women [1]. CRC is the major cause of cancer-related death worldwide, approximately more than 70000 deaths and nearly 1.4 million new cases per year [2]. Recent years, CRC incidence and mortality rates have been found to rapidly rise in Asian countries, especially among people aged 30-50[2,3]. CRC is a heterogeneous disease in which environmental, genetic, and lifestyle factors are involved. CRC is a malignant tumor that originates from the intestine mucosal epithelium, mostly developed from adenomatous polyps or adenomas [4, 5]. The survival rate of CRC patients have increased over the past few years due to earlier diagnosis and advanced treatment methods, though, cure rate is still low. Therefore, it's an imperative need for investigating new prognostic factor or molecular target in CRC.

Recently, gene microarray, high-throughput sequencing and bioinformatics analysis have been broadly applied in cancer research. Microarray analysis allows simultaneous investigation of several thousands of cancer-related and cancer-specific genes [6], which can improve the understanding of molecular mechanism of cancers. Many studies showed that some important genes have been found in cancers by applying gene microarray and bioinformatics [7]. Sun TT et al, have found that IncRNA GClnc1 promotes gastric carcinogenesis by using the human lncRNA array [8]. Bonavida B et al, have showed that YY1 could be considered as a potential novel therapeutic target in human cancers by bioinformatics analysis [9]. Thus, in this study, we applied bioinformatics and gene expression datasets to discovery DEGs in CRC. Among these DEGs, we further identified that MCM7 could be considered as a key hub gene which was overexpressed in CRC tissues compared with non-cancerous tissues by using bioinformatics methods.

The minichromosome maintenance (MCM) complex is composed of six highly conservative MCM proteins, named MCM2-7(small body maintenance complex component 2-7), which is necessary to initiate DNA replication. MCM is a marker associated with proliferation of several types of cancer $[6,10]$. MCM2-7 forms the pre-replication complex (pre-RC), and then recognizes the origin recognition complex (ORC) before DNA replication [11]. MCM7 is one of the important components of DNA replication complex. However, the pathogenesis of MCM7 remains unclear in CRC.

In this study, we investigated MCM7 was up-regulated in CRC tissue compared with normal tissue. Additionally, we evaluated the clinical significance of MCM7 and the prognostic in CRC patients. 


\section{Materials and Methods}

\section{Gene expression data from GEO and TCGA}

The gene expression datasets of GSE21510 and GSE103512 were obtained from GEO. GSE21510 contains 123 CRC tissues and 25 normal tissues. GSE103512 includes 57 CRC samples with 12 matched normal samples. Another, CRC mRNA expression profile was downloaded from TCGA, which includes 519 samples.

\section{Data processing and DEGs screening}

The R package 'edgeR' and'limma' were applied to identify the DEGs between CRC tissues and normal tissues. The inclusion standard of DEGs from TCGA was adjusted $P<0.01$ and log2 Fold Change $(\mathrm{FC})>2$. DEGs from GEO datasets were selected with the criterion of combined adjusted $p<0.01$ and $\log 2 \mathrm{FC}>1$ or $\log 2 \mathrm{FC}>0.58$.

\section{Protein-protein interaction (PPI) network construction and module analysis}

STRING (https://string-db.org) was applied to construct PPI network for DEGs with confidence score $>0.4$ and $p<0.01$. Cytoscape software was used to visualize network. Another, the Molecular Complex Detection (MCODE) app was used to screen modules of PPI network in Cytoscape under degree cutoff $=2$, node score cutoff $=0.2$, $\mathrm{k}$-core $=2$, and max. $\mathrm{depth}=100$. Moreover, the nodes were calculated the degree value.

\section{Clinical samples and tissue microarray}

Twenty-two pairs of primary CRC samples with matched adjacent non-tumorous samples were obtained from specimens' library of the second Affiliated Hospital of Xi'an Jiaotong University. Every patients signed the informed consent, and the Clinical Research Ethics Committee of the second Affiliated Hospital of Xi'an Jiaotong University approved the ethics.

Tissue microarray (HCoIA180Su15) was purchased from Shanghai Outdo Biotech (China) which contains 101 CRC samples and 79 adjacent non-tumorous samples. The tissues collection time was from April 2008 to November 2008, and the follow-up interval was 6.7 to 7.2 years.

\section{Immunohistochemistry}

Paraffin-embedded microarray was dewaxed in xylene and gradient ethanol. Antigen repair was performed by microwave. The slide was incubated with 3\% hydrogen peroxide for 10 mins to remove endogenous catalase activity, and then incubated with goat serum at room temperature for one hour. Then slice was incubated with the anti-MCM7 (1:5000, Abcam, ab52489) at $4^{\circ} \mathrm{C}$ overnight. Anti-lgG (1:400) was co-incubated with section at room temperature for $1 \mathrm{~h}$, then incubated with streptomycin HRP for 30 minutes, stained with DAB, and then counterstained with haematoxylin. After dehydrating with gradient alcohol and dimethylbenzene, seal the sheet with neutral resin.

\section{Evaluation of immunostaining intensity}

Tissue microarray was scored independently by two pathologists blinded to the clinicopathologic features and outcomes of patients. The protein expression level was calculated and classified based on the percentage of positive cells and the intensity of staining. The immunoreactivity was divided into 5 grades according to the percentage of stained cells: no staining (0), $1-25 \%$ (1), $26-50 \%$ (2), $51-75 \%$ (3) and $>75 \%$ (4). The intensity of staining was divided into four grades (intensity scores): negative (0), weak (1), moderate (2) and strong (3). Finally, the overall score of histological was multiplication of two values. The score of $0-12$ was calculated and graded as low (score: $0-4$ ) or high (score: 5-12).

\section{Western Blotting analysis}

Western Blotting was performed as previously described [12]. The primary antibody against MCM7 was purchased from Abcam.

\section{Statistical analysis}

Prism 6 (San Diego, CA, USA) and SPSS 22.0 (Chicago, IL, USA) were applied in this study. We tested the differences between two groups whether the data conforms to the test of homogeneity of variance and the test of normal distribution before performing two-tail Student's $t$ test. Overall survival was assessed by KaplanMeier analysis and log-rank test. The relationship between the expression of MCM7 and the clinicopathological features in CRC was analyzed by chi-square test. Univariate and Multivariate Cox regression proportional hazards analysis were used to assess whether the gene expression and other clinicopathologic characteristics are an independent prognostic factor. $P<0.05$ was considered statistically significant. All values are expressed by means of SD.

\section{Results}

\section{The screening of DEGs in CRC multiple datasets}

To screen some important colorectal cancer-related genes, we analyzed mRNA microarrays by bioinformatics methods. Gene expression datasets about CRC (GSE21510, GSE103512)

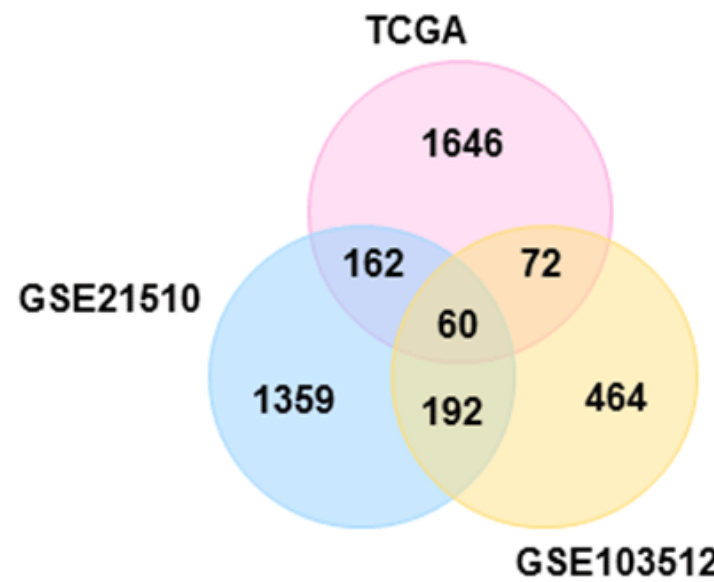

Figure 1: Venn diagram showing up-regulated genes in three datasets 
were obtained from GEO database. Another mRNA expression of CRC was obtained from TCGA. Three datasets were analyzed by "limma" and "edgeR" package of R language. According to the selection criteria, we found that 1940 genes were up-regulated in CRC tissues compared with normal tissues from TCGA. Another, 1773 mRNAs were up-regulated in GSE21510 and 788 mRNAs were increased in GSE103512. Then a total of 60 DEGs which were up-regulated in CRC tissues compared with normal tissues were identified in all three datasets (Figure 1).

The construction of PPI network and the selection of hub genes

60 DEGs were submitted to the STRING database, and then a PPI network was constructed which contained 34 nodes and 104 edges (Figure 2A). We analyzed the PPI network by applying MCODE topology algorithm of Cytoscape software, one core cluster was identified in network which included 8 hub genes (Figure 2B). Moreover, we calculated the degree value of each gene in PPI network. PAICS, MCM2, BIRC5, MCM7, AURKA, CSE1L, RAN, PRC1, PTTG1, ATAD2 were the top ten genes with high degree (Figure 2C). Based on MCODE and degree value analysis, we chose seven genes (MCM2, BIRC5, MCM7, AURKA, PRC1, PTTG1, and ATAD2) as hub genes. Ultimately, combined with bioinformatics analysis and previously studies, it was found that the pathogenesis of MCM7 remains unclearly in CRC.

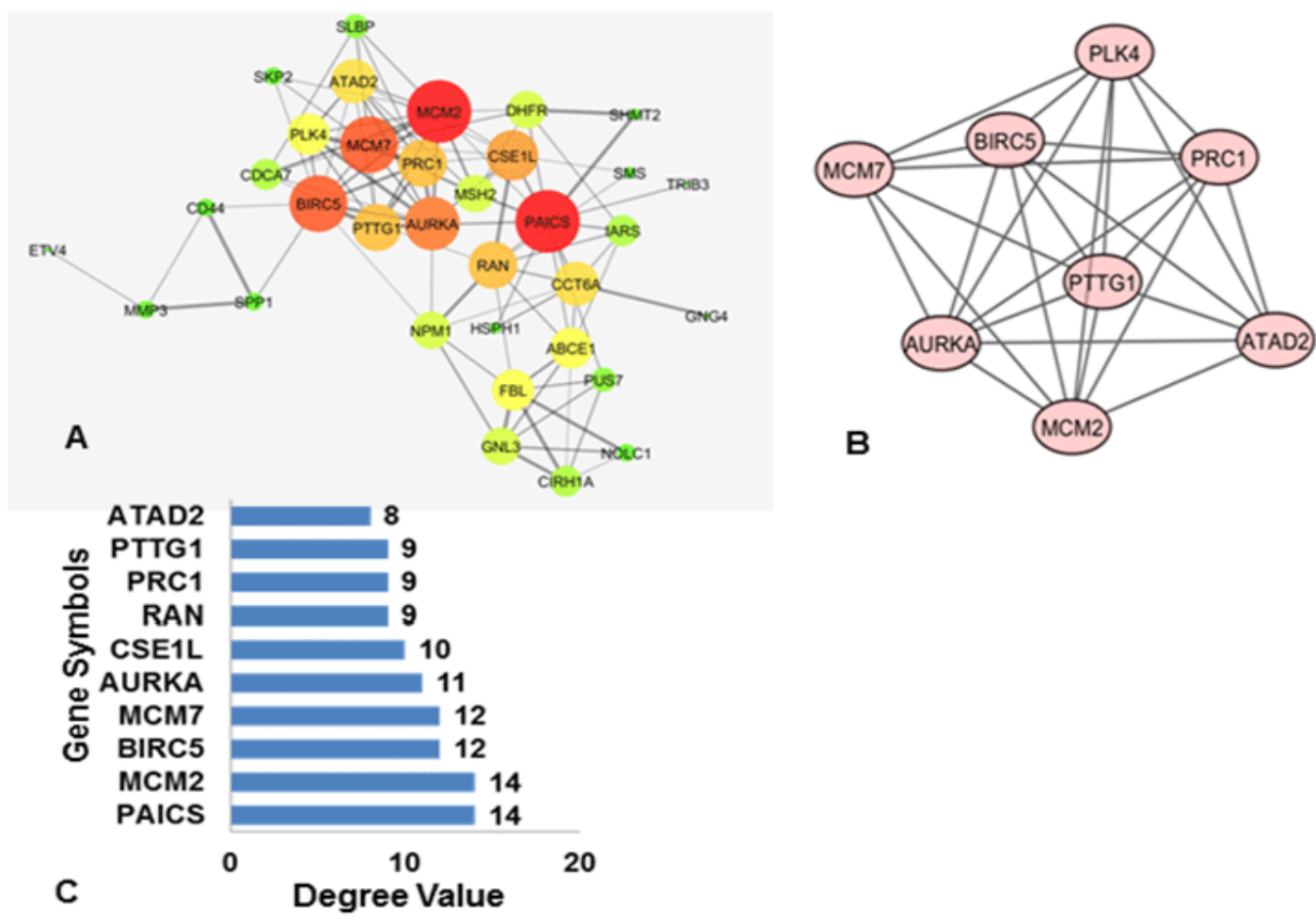

Figure 2: (A) The protein -protein interaction network of 60 DEGs. (B) The PPI network of 8 hub genes calculated by MCODE. (C)Top ten degree genes of DEGs

\section{MCM7 is up regulated in human CRC patients}

We further detected that the expression level of MCM7 in multiple datasets. The mRNA expression of MCM7 was significantly up regulated in 70 CRC samples and 12 noncancerous samples from GSE9348 (Figure 3A). In TCGA including $478 \mathrm{CRC}$ tissues and 42 non-tumorous tissues, we also found that the mRNA level of MCM7 was increased (Figure 3B). Then, we examined the protein expression of MCM7 in 6 pairs of human CRC tissues and the adjacent non-tumor tissues. As expected, MCM7 protein expression was up-regulated in CRC tumor tissues compared to the adjacent non-tumor tissues as determined by Western Blotting analysis (Figure 3C, $p<0.01$ ). Consistently, immunohistochemical (IHC) staining of the human CRC tissue microarray revealed that MCM7 was predominantly localized to both the cytoplasm and nucleus and was significantly upregulated in CRC tissues compared with that in normal tissues $(p<0.001)$ (Figure 3D and Table 1).

\section{The correlation between MCM7 and clinical characteristic} features

Our work demonstrated that MCM7 was increased in CRC. We further analyzed the correlation between the expression of MCM7 and characteristic features, and found that higher expression of MCM7 was positively associated with lymph node metastasis and TNM stage ( $p<0.05$, Table 2$)$. However, upregulation of MCM7 in CRC tissues was not correlated with age, gender, tumor location, or size ( $p>0.05$, Table 2 ). 

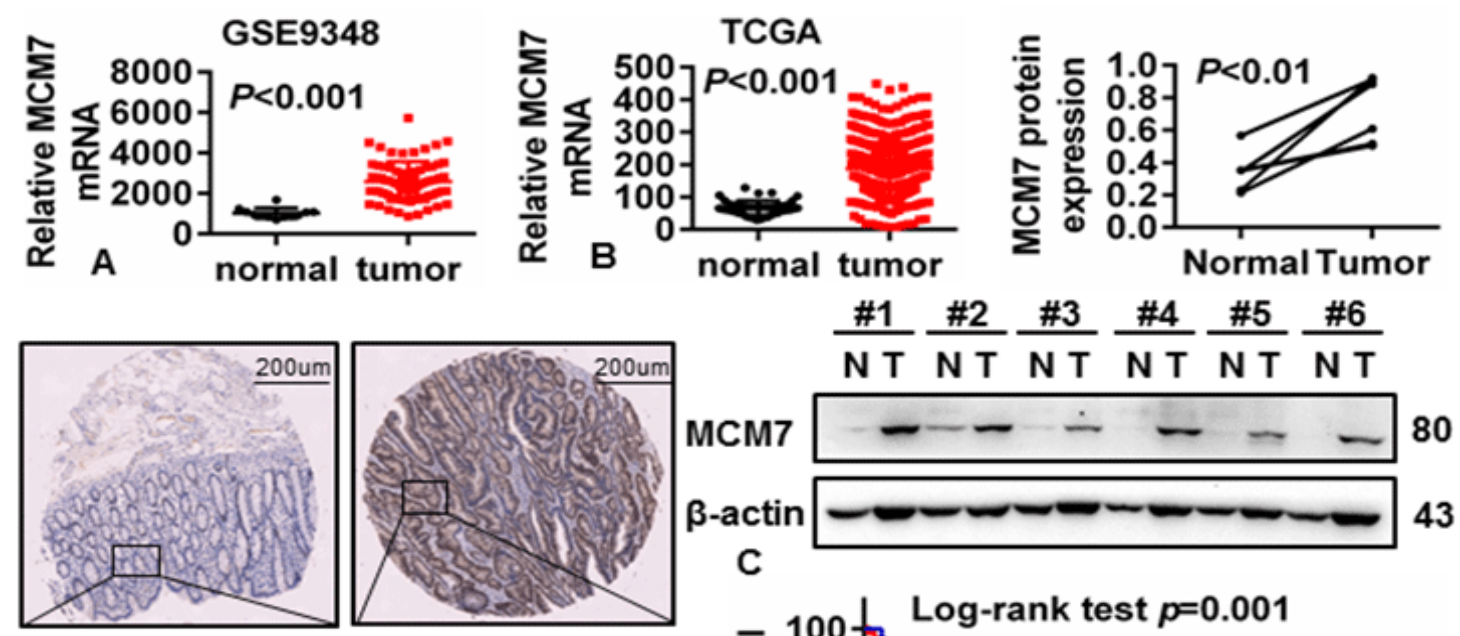

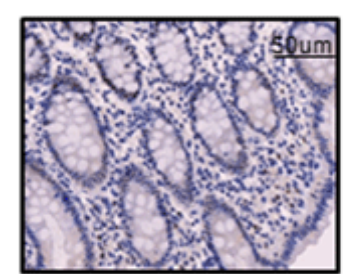

Normal Tissues

D

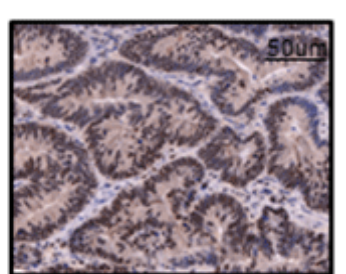

Tumor Tissues

Figure 3: The mRNA level of MCM7 in GSE9348 (A) and TCGA (B). (C) The protein expression of MCM7 in human CRC tissues. (D) The immunohistochemical staining of MCM7 by using tissues microarray. (E) Correlations of MCM7 expression level with OS in CRC patients by Kaplan-Meier analysis

Table 1: The MCM7 level in CRC tissue microarray

\begin{tabular}{|c|c|c|c|c|}
\hline Group & Cases & MCM7 level & \multicolumn{2}{|c|}{ high } \\
\hline & & low & 9 & $<0.001$ \\
\hline Normal Tissues & 79 & 70 & 67 & \\
\hline Tumor Tissues & 101 & 34 & 67 \\
\hline
\end{tabular}

Table 2: Association of MCM7 level with clinicopathological parameters of patients with CRC

\begin{tabular}{|c|c|c|c|c|}
\hline \multirow{2}{*}{ Category } & \multirow{2}{*}{$\mathbf{n}$} & \multicolumn{2}{|c|}{ MCM7 } & \multirow{2}{*}{$P$} \\
\hline & & low expression & high expression & \\
\hline Gender & & & & 0.986 \\
\hline male & 56 & 19 & 37 & \\
\hline female & 44 & 15 & 29 & \\
\hline Age & & & & 0.16 \\
\hline$<60$ & 21 & 10 & 11 & \\
\hline$\geq 60$ & 74 & 23 & 51 & \\
\hline Tumor Size & & & & 0.559 \\
\hline$<5$ & 38 & 14 & 24 & \\
\hline$\geq 5$ & 61 & 19 & 42 & \\
\hline Location & & & & 0.156 \\
\hline ascending colon & 45 & 12 & 33 & \\
\hline transverse colon & 11 & 2 & 9 & \\
\hline
\end{tabular}

Citation: Lei D, Ting W, Bing X, Jing Z, et al. (2019) Up-Regulated Expression of MCM7 is a Poor Prognostic Factor in Colorectal Cancer. 


\begin{tabular}{|c|c|c|c|c|}
\hline descending colon & 13 & 7 & 6 \\
\hline sigmoid colon & 32 & 13 & 35 & \\
\hline Lymph node metastasis & & & 31 & $<0.05$ \\
\hline negative & 61 & 26 & 3 & $<0.05$ \\
\hline positive & 39 & 8 & 32 & \\
\hline AJCC stage & 5 & 2 & 28 & \\
\hline I & 56 & 24 & 3 & \\
\hline II & 36 & 8 & & \\
\hline III & 3 & 0 & & \\
\hline
\end{tabular}

\section{The Relationship Between MCM7 Expression And Prognosis}

We next revealed the relationship between MCM7 expression and the prognosis of CRC. Patients with higher expression of MCM7 showed shorter overall survival than those with lower MCM7 expression (Figure 3E). Moreover, univariate and multivariate COX regression analysis were used to confirm whether MCM7 can be used as an independent factor for poor prognosis of CRC. Univariate regression analysis showed that lymph node metastasis, TNM stage, and MCM7 expression were associated with shorter survival time $(p<0.001$, Table 3$)$. Multivariate analysis further demonstrated that the expression level of MCM7 was an independent risk factor for a poor prognosis in CRC patients (Table 4).

Table 3: Univariate analysis of the correlation between clinicopathological features and survival of patients in CRC

\begin{tabular}{|c|c|c|c|c|c|c|}
\hline \multirow{2}{*}{ Category } & \multicolumn{2}{|c|}{ Cumulative survival rates (\%) } & \multirow{2}{*}{$\begin{array}{l}\text { Mean survival time } \\
\text { (mo) }\end{array}$} & \multirow{2}{*}{ Hazard ratio } & \multirow{2}{*}{$\begin{array}{c}\text { 95\% Confidence } \\
\text { interval }\end{array}$} & \multirow{2}{*}{$p$} \\
\hline & 3-Years & 5-Years & & & & \\
\hline \multicolumn{7}{|l|}{ Gender } \\
\hline male & 62.5 & 50 & 63.8 & 1.038 & $0.619-1.740$ & 0.888 \\
\hline female & 59.1 & 52.3 & 64.7 & & & \\
\hline \multicolumn{7}{|l|}{ Age } \\
\hline$<60$ & 71.4 & 61.9 & 75.2 & 0.545 & $0.267-1.110$ & 0.094 \\
\hline$\geq 60$ & 56.8 & 45.9 & 59 & & & \\
\hline \multicolumn{7}{|l|}{ Tumor Size } \\
\hline$<5$ & 73.7 & 52.6 & 69.3 & 0.883 & $0.491-1.412$ & 0.497 \\
\hline$\geq 5$ & 52.5 & 49.2 & 60.3 & & & \\
\hline \multicolumn{7}{|l|}{ Location } \\
\hline ascending colon & 51.1 & 46.7 & 54.9 & & & 0.117 \\
\hline transverse colon & 54.5 & 36.4 & 54.5 & 2 & $1.082-3.697$ & 0.027 \\
\hline descending colon & 61.5 & 53.8 & 69.3 & 1.808 & $0.736-4.400$ & 0.196 \\
\hline sigmoid colon & 75 & 59.4 & 76.4 & 1.08 & $0.419-2.784$ & 0.873 \\
\hline \multicolumn{7}{|c|}{ Lymph node metastasis } \\
\hline negative & 77 & 63.9 & 76.3 & 0.383 & $0.229-0.642$ & $<0.001$ \\
\hline positive & 35.9 & 30.8 & 44.6 & & & \\
\hline \multicolumn{7}{|l|}{ AJCC stage } \\
\hline I & 80 & 80 & 98.4 & & & $<0.001$ \\
\hline II & 75 & 60.7 & 74 & 0.014 & $0.001-0.149$ & $<0.001$ \\
\hline III & 38.9 & 33.3 & 47.6 & 0.046 & $0.012-0.174$ & $<0.001$ \\
\hline IV & 0 & 0 & 8 & 0.103 & $0.028-0.383$ & $<0.001$ \\
\hline \multicolumn{7}{|l|}{ MCM7 level } \\
\hline low & 91.2 & 85.3 & 93.4 & 0.156 & $0.071-0.345$ & $<0.001$ \\
\hline high & 44.8 & 32.8 & 48.5 & & & \\
\hline
\end{tabular}




\begin{tabular}{|c|c|c|c|}
\hline Category & Hazard ratio & 95\% Confidence interval & $p$ \\
\hline \multicolumn{4}{|l|}{ Tumor Size } \\
\hline$<5$ & 1.531 & $0.892-2.627$ & 0.122 \\
\hline \multicolumn{4}{|l|}{$\geq 5$} \\
\hline \multicolumn{4}{|c|}{ Lymph node metastasis } \\
\hline negative & 2.611 & $1.532-4.450$ & $<0.001$ \\
\hline \multicolumn{4}{|l|}{ positive } \\
\hline \multicolumn{4}{|l|}{ MCM7 level } \\
\hline low & 0.167 & $0.075-0.371$ & $<0.001$ \\
\hline high & & & \\
\hline
\end{tabular}

\section{Discussion}

Gene chip is a kind of microarray technology which could obtain high-throughput gene expression information rapidly. It can not only be used as a new method to study tumor-related molecular mechanisms, but also be used for the prediction of clinical molecular markers. At present, due to the differences among different platforms, different samples and different researchers, in order to increase the reliability of data, this study selected three data sets and used bioinformatics to analyze datasets. We found that 60 genes were highly expressed in the three datasets compared with normal tissues. Further, we identified 7 hub genes which may be related to the progression of CRC through MCODE and degree values analysis. The seven hub genes contain MCM2, BIRC5, MCM7, AURKA, PRC1, PTTG1, and ATAD2. Coos JA et al [13], found that increased the expression of AURKA was associated with poor prognosis in patients with liver metastasis of CRC. Li PL et al, found that microRNA-218 as a prognostic indicator enhanced 5-fluorouracil-induced apoptosis by targeting BIRC5 in colorectal cancer [14]. PTTG1 promoted CRC growth and metastasis as an independent prognostic factor [15]. Hou $\mathrm{M}$ et al investigated that ATAD2 overexpression is associated with progression and prognosis in CRC [16]. Many papers have reported that MCM2 was involved in the progression of various tumors. These literatures verified that the bioinformatics analysis could be as a new method to discover tumor-related genes. Currently, there is still lack of previous studies on the relationship between MCM7 and CRC. Though, many studies have shown that MCM7 proteins are involved in tumorigenesis [10]. MCM7 regulated the proliferation and migration of esophageal squamous cell carcinoma cells by activating the AKT1/mTOR pathway [11]. Li J et al, showed that knockdown of MCM7 expression could promote the apoptosis of RB-deficient tumor cells [17].

The role of MCM7 in leukemia has also been reported. The polymorphism of MCM7 was related to the recurrence and overall survival rate of acute myeloid leukemia, which could predict the prognosis of acute myeloid leukemia [18]. Tian L et al found that knockdown of MCM7 inhibited the proliferation of K562 cells and promoted cell apoptosis [19].Kim SH et al found that MCM7 was related to cisplatin resistance in bladder cancer by bioinformatics analysis of gene chip [20].
To further verify the expression of MCM7 in CRC, we explored that the mRNA level of MCM7 was increased in CRC samples compared with normal samples by using gene expression data. Additionally, the protein level of MCM7 was up-regulated in human CRC tissues and tissues microarray. Furthermore, we detected that MCM7 overexpression was statistically correlative with lymph node metastasis and TNM stage, indicating that MCM7 might promote metastasis. Kang W et al reported that the increased expression of MCM7 in gastric cancer could promote cell proliferation and invasion, and induce apoptosis as a prognostic marker [21]. Further, higher expression of MCM7 showed shorter survival time. Univariate and multivariate regression analyses investigated that MCM7 could be a poor prognostic biomarker for CRC. Qu K et al found that MCM7 promoted hepatocellular carcinoma progression through cyclin D1-dependent signaling as a prognostic marker for patients [10].Liu YZ et al found that MCM7 may be a potential prognostic marker in non-small cell lung cancer [22]. It has also been reported that MCM7 was a prognostic marker of esophageal cancer [23], endometrial cancer [24] and pituitary adenoma [25]. We have demonstrated that high expression of MCM7 is an independent poor prognostic factor in CRC. We further plan to design experiments in vivo and in vitro to further explore the pathogenesis of MCM7 in CRC.

\section{Conclusion}

In our study, the mRNA and protein level of MCM7 were upregulated in CRC tissues. In addition, the high level of MCM7 was correlated with TNM stage and lymph node metastasis. Furthermore, we demonstrated the expression of MCM7 could be an independent prognostic factor for CRC, which revealed the important role of MCM7 in CRC.

\section{Acknowledgements}

This work was supported by Key research and development program of Shaanxi province under grant (2018ZDXMSF-055); Central government guide the development of local science and technology special funds under grant (2016ZY-HM-01).

\section{References}

1. Siegel RL, Miller KD, Fedewa SA, Ahnen DJ, Meester RGS, Barzi A, et al. Colorectal cancer statistics, 2017. CA Cancer J Clin. 2017;67(3):177193. doi: $10.3322 /$ caac. 21395 
2. Arnold M, Sierra MS, Laversanne M, Soerjomataram I, Jemal A, Bray F. Global patterns and trends in colorectal cancer incidence and mortality. Gut. 2017;66(4):683-691. doi: 10.1136/gutjnl-2015-310912

3. Center MM, Jemal A, Smith RA, Ward E. Worldwide variations in colorectal cancer. CA Cancer J Clin. 2009;59(6):366-378. doi: 10.3322/ caac. 20038

4. Aran V, Victorino AP, Thuler LC, Ferreira CG. Colorectal cancer: epidemiology, disease mechanisms and interventions to reduce onset and mortality. Clin Colorectal Cancer.2016;15(3):195-203. doi: 10.1016/j.clcc.2016.02.008

5. JS Levine, DJ Ahnen. Adenomatous polyps of the colon. N Engl J Med. 2006;355:2551-2557. doi: 10.1056/NEJMcp063038

6. Gutiérrez ML, Corchete L, Teodosio C, Sarasquete ME, del Mar Abad $\mathrm{M}$, Iglesias $\mathrm{M}$, et al. Identification and characterization of the gene expression profiles for protein coding and non-coding RNAs of pancreatic ductal adenocarcinomas. Oncotarget. 2015;6(22):1907019086. doi: 10.18632/oncotarget. 4233

7. Sun TT, He J, Liang Q, Ren LL, Yan TT, Yu TC, et al. LncRNA GClnc1 Promotes Gastric Carcinogenesis and May Act as a Modular Scaffold of WDR5 and KAT2A Complexes to Specify the Histone Modification Pattern. Cancer Discov. 2016;6(7):784-801. doi: 10.1158/2159-8290

8. Bonavida B, Kaufhold S. Prognostic significance of YY1 protein expression and mRNA levels by bioinformatics analysis in human cancers: a therapeutic target. Pharmacol Ther. 2015;150:149-168. doi: 10.1016/j.pharmthera.2015.01.011

9. Kai Qu, Zhixin Wang, Haining Fan, Juan Li, Jie Liu, Pingping Li, et al. MCM7 promotes cancer progression through cyclin D1dependent signaling and serves as a prognostic marker for patients with hepatocellular carcinoma. Cell Death Dis. 2017;8(2): e2603. doi:10.1038/cddis.2016.352

10. Qiu YT, Wang WJ, Zhang B, Mei LL, Shi ZZ. MCM7 amplification and overexpression promote cell proliferation, colony formation and migration in esophageal squamous cell carcinoma by activating the AKT1/mTOR signaling pathway. Oncol Rep. 2017;37(6):3590-3596. doi: 10.3892/or.2017.5614

11. Liu X, Hu Z, Zhou B, Li X, Tao R. Chinese Herbal Preparation Xuebijing Potently Inhibits Inflammasome Activation in Hepatocytes and Ameliorates Mouse Liver Ischemia-Reperfusion Injury. PLoS One. 2015;10:e0131436. Doi:10.137/journal.phone.0131436

12. Goos JA, Coupé VM, van de Wiel MA, Diosdado B, Delis-Van Diemen $\mathrm{PM}$, Hiemstra AC, et al. A prognostic classifier for patients with colorectal cancer liver metastasis, based on AURKA, PTGS2 and MMP9. Oncotarget. 2016;7(2):2123-2134. doi: 10.18632/oncotarget.6188
13. Li PL, Zhang X, Wang LL, Du LT, Yang YM, Li J, et al. MicroRNA-218 is a prognostic indicator in colorectal cancer and enhances 5-fluorouracil-induced apoptosis by targeting BIRC5. Carcinogenesis. 2015;36(12):1484-1493. doi: 10.1093/carcin/bgv145

14. Ren $\mathrm{Q}$ Jin B. The clinical value and biological function of PTTG1 in colorectal cancer. Biomed Pharmacother. 2017;89:108-115. doi: 10.1016/j.biopha

15. Hou M, Huang R, Song Y, Feng D, Jiang Y, Liu M. ATAD2 overexpression is associated with progression and prognosis in colorectal cancer. Jpn J Clin Oncol. 2016;46(3):222-227. doi: 10.1093/jjco/hyv195

16. Li J, Liu J, Liang Z, He F, Yang L, Li P, et al. Simvastatin and Atorvastatin inhibit DNA replication licensing factor MCM7 and effectively suppress RB-deficient tumors growth. Cell Death Dis. 2017;8(3):e2673. doi: 10.1038/cddis.2017.46

17. Lee JS, Cheong HS, Koh Y, Ahn KS, Shin HD, Yoon SS, et al. MCM7 polymorphisms associated with the AML relapse and overall survival. Ann Hematol. 2017;96(1):93-98. doi: 10.1007/s00277-016-2844-2

18. Tian L, Liu J, Xia GH, Chen BA. RNAi-mediated knockdown of MCM7 gene on CML cells and its therapeutic potential for leukemia. Med Oncol. 2017;34(2):21. doi: 10.1007/s12032-016-0878-x

19. Kim SH, Ho JN, Jin H, Lee SC, Lee SE, Hong SK, et al. Upregulated expression of BCL2, MCM7, and CCNE1 indicate cisplatin-resistance in the set of two human bladder cancer cell lines: T24 cisplatin sensitive and T24R2 cisplatin resistant bladder cancer cell lines. Investig Clin Urol. 2016;57(1):63-72. doi: 10.4111/icu.2016.57.1.63

20. Kang W, Tong JH, Chan AW, Cheng AS, Yu J, To K. MCM7 serves as a prognostic marker in diffuse-type gastric adenocarcinoma and siRNA-mediated knockdown suppresses its oncogenic function. Oncol Rep. 2014;31(5):2071-2078. doi: 10.3892/or.2014.3094

21. Liu YZ, Jiang YY, Hao JJ, Lu SS, Zhang TT, Shang L, et al. Prognostic significance of MCM7 expression in the bronchial brushings of patients with non-small cell lung cancer (NSCLC). Lung Cancer. 2012;77(1):176-182. doi: 10.1016/j.lungcan

22. Zhong X, Chen X, Guan X, Zhang H, Ma Y, Zhang S, et al. Overexpression of G9a and MCM7 in oesophageal squamous cell carcinoma is associated with poor prognosis. Histopathology. 2015;66(2):192-200. doi: 10.1111/his.12456

23. Li SS1, Xue WC, Khoo US, Ngan HY, Chan KY, Tam IY, et al. Replicative MCM7 protein as a proliferation marker in endometrial carcinoma: a tissue microarray and clinicopathological analysis. Histopathology. 2005;46(3):307-313. doi: 10.1111/j.1365-2559.2005.02069

24. Coli A, Asa SL, Fadda G, Scannone D, Chiloiro S, De Marinis L, et al. Minichromosome maintenance protein 7 as prognostic marker of tumor aggressiveness in pituitary adenoma patients. Eur J Endocrinol. 2016;174(3):307-314. doi: 10.1530/EJE-15-0586 